Revue d'histoire de l'Amérique française

REVUE D.HISTOIRE DE L'AMÉRIQUE FRANÇAISE

\title{
Un patriote inconnu : Joseph-Édouard Turcotte
}

\section{Louisette Pothier}

Volume 38, numéro 1, été 1984

URI : https://id.erudit.org/iderudit/304237ar

DOI : https://doi.org/10.7202/304237ar

Aller au sommaire du numéro

Éditeur(s)

Institut d'histoire de l'Amérique française

ISSN

0035-2357 (imprimé)

1492-1383 (numérique)

Découvrir la revue

Citer cette note

Pothier, L. (1984). Un patriote inconnu : Joseph-Édouard Turcotte. Revue

d'histoire de l'Amérique française, 38(1), 59-71. https://doi.org/10.7202/304237ar d'utilisation que vous pouvez consulter en ligne.

https://apropos.erudit.org/fr/usagers/politique-dutilisation/ 
NOTE DE RECHERCHE

\title{
UN PATRIOTE INCONNU: JOSEPH-ÉDOUARD TURCOTTE
}

\author{
LOUISETTE POTHIER \\ Séminaire de Sherbrooke
}

Même après la parution de l'oeuvre de Jean-Paul Bernard, qui veut faire le point sur les Rébellions de 1837-18381, tout n'est évidemment pas dit sur le sujet. L'auteur le reconnaît lui-même. Il écrit qu'il veut «proposer un outil de travail commode et, en même temps, montrer qu'il y a encore beaucoup à faire sur la question $»^{2}$. Pour les années qui précèdent immédiatement les Troubles, on connaît assez bien l'action et le discours des chefs, plus particulièrement de ceux qui siègent à la Chambre d'Assemblée du Bas-Canada. Pour la période des Troubles, on a le récit des actions des Patriotes qui sont mêlés aux engagements. Pour la répression qui suit, on identifie dans bon nombre de leurs traits ceux qui ont été poursuivis par la justice. Mais dans l'historiographie sur les événements, les revendications et les personnages de premier plan liés aux Rébellions, il y a encore place pour la découverte et l'interprétation. Si l'on connaît assez bien les chefs, les braves et «les martyrs», que sait-on des Patriotes «ordinaires», ceux qui n'ont été ni député ni stratège, ceux qui n'habitaient ni Saint-Charles, ni SaintDenis, ni Saint-Benoît, ceux qui n'ont pas eu «la chance» de tomber entre les mains de la justice et d'accéder à la renommée? Ces Patriotes «ordinaires» échappent encore à l'histoire.

Un certain nombre d'entre eux figurent sur les listes dressées par les historiens, dont la plus récente et la plus complète est celle que vient de publier Jean-Paul Bernard ${ }^{3}$. Des 8000 personnes qu'on pourrait qualifier de Patriotes, Bernard en a repéré 2 100. C'est déjà un acquis. Mais même de ces 2100 , que sait-on?

Au cours de recherches sur un homme politique du XIX siècle, j'ai découvert une «période patriote» dans la vie de Joseph-Édouard

\footnotetext{
1 Jean-Paul Bernard, Les Rébellions de 1837-1838, Les patriotes du Bas-Canada dans la mémoire collective et chez les historiens (Montréal, Boréal Express, 1983), 351 p.

2 Ibid., 8.

3 Ibid., 285-325.
} 
Turcotte ${ }^{4}$. Dans la liste de Bernard, il s'appelle Joseph Turcotte, il a 29 ans, il habite Québec, sa profession est inconnue ${ }^{5}$. Il s'agit indiscutablement de Joseph-Édouard Turcotte. Celui-ci a effectivement 29 ans et il est avocat à Québec au moment des Troubles de 1837. Né à Gentilly en 1808, il est le cinquième enfant de Joseph Turcot, marchand, et de Marguerite Marchildon. Il est devenu ecclésiastique, professeur de Belles-Lettres au Séminaire de Nicolet en 1829-1830 et titulaire de la classe de rhétorique au Collège de Sainte-Anne-de-la-Pocatière en 1830-1831. Au moment où la situation devient tendue à la Chambre et dans la rue, entre 1832 et 1834 , Turcotte vit à Québec. Revenu à l'état laïque, il fait sa «cléricature» à l'étude d'Elzéar Bédard et est copiste à la Chambre. Il a donc l'occasion de fréquenter de près les chefs Patriotes et d'épouser leurs idées et leur cause. La fièvre monte alors dans le pays et la résistance se radicalise. Les positions se durcissent, les griefs se multiplient, l'impasse s'annonce à la Chambre; Papineau et ses lieutenants préparent les Quatre-vingt-douze Résolutions, tandis que s'affirment les modérés sous la direction d'Elzéar Bédard et qu'apparaissent les premières dissensions dans le mouvement. Le premier engagement de Turcotte pour la cause papineauiste remonte à cette période d'effervescence. Elle prend la forme de poèmes politiques qui sont publiés dans les journaux sous les seules initiales J.E.T., et qui seront reproduits sous son nom complet dans un ouvrage qui lui est contemporain, Le répertoire national ${ }^{6}$. L'identité du poète est aussi confirmée dans une lettre de Thomas Fortier, médecin à Gentilly, à Ludger Duvernay, directeur de la Minerve ${ }^{7}$.

Un premier poème, sans titre, est publié dans le journal la Minerve du 30 décembre $1833^{8}$. L'auteur le présente comme «un faible tribut» offert aux «constants efforts» des Patriotes «dans la cause commune»; essentiellement, c'est un appel à l'émancipation du peuple canadien. Un second poème, publié dans la Minerve du 26 mai 1834, a pour titre «L'Anniversaire du grand meurtre» ${ }^{9}$. Il rappelle le décès de trois Montréalais abattus par l'armée britannique dans une émeute occasionnée

\footnotetext{
4 Joseph-Édouard Turcotte (1808-1864) est connu comme un homme politique (1841-1844, 1851-1864), maire de Trois-Pivières (1857-1863), co-fondateur du Séminaire de Trois-Rivières (1860), entrepreneur dans $\|^{\prime}$ exploitation des Forges Radnor et dans différents travaux publics pour la mise en valeur de Trois-Rivières et de la région.

5 Jean-Paul Bernard, op. cit., 312.

6 J. Huston éd., Le répertoire national ou recueil de littérature canadienne (1 ère éd., Montréal, Lovell \& Gibson, 1848), I: 239, 240, 330, 342.

Fortier écrit: L'écrit en vers, signé J.E.T., est l'ouvrage du fils du Capt. Turcot que tu as fort bien connu dans ton temps et le frère de Narcisse Turcotte des Trois-Rivières qui te transmet des écrits. Ce sont mes jeunes amis. Il y a longtemps que je les incite à écrire. Joseph est maintenant à Québec (ANQ, Papiers Duvernay, f. 204).

Reproduit dans J. Huston, éd., op. cit., 1 êre éd., I: 239.

9 Dans J. Huston, éd., op. cit., $2^{\mathrm{e}}$ éd., I: 282.
} 
par l'élection du 30 mai 1832 dans le quartier ouest de Montréal ${ }^{10}$. Le poète saisit l'occasion d'appeler les Canadiens à se libérer, eux dont la conscience nationale commence à s'éveiller. Le troisième poème, "À l'Hon. L. J. Papineau», est d'abord publié dans la Minerve du 24 juillet 1834 , puis reproduit dans le Canadien des 30 juillet et $1^{\mathrm{er}}$ août suivants ${ }^{11}$. Le contexte politique dans lequel ce poème est publié permet de situer Turcotte dans le groupe des Patriotes radicaux. Depuis que Bédard et les députés de la région de Québec ont refusé de boycotter les travaux de la Chambre, il y a, malgré le rapprochement apparent autour des Quatre-vingt-douze Résolutions, deux clans de Patriotes. La rupture ne viendra qu'en 1835, mais elle est déjà bien amorcée de sorte que cet hommage de Turcotte à Papineau est sans équivoque. Papineau y est comparé aux plus grands libérateurs de la Bible, à Moïse, au Christ même, tandis que les «modérés», ses proches qui l'abandonnent, sont comparés au peuple juif qui manque à sa mission et se refuse au salut:

De leur joug ta main nous délivre,

Et nous avons comme au grand livre,

Nos docteurs de l'ancienne loi;

Dans leur tendre sollicitude,

Et pour sauver la multitude,

Criant: Il veut se faire roi!

À nos frères qui t'abandonnent,

Quand tes prodiges les étonnent:

Qui près de recevoir encor

La Table à ta vertu commise

Et près de la terre promise

Vont sacrifier au veau d'or...

Un dernier poème s'intitule «Le retour». Il est dédié à Augustin-Norbert Morin qui rentre d'Angleterre où il a présenté les Quatre-vingt-douze Résolutions ${ }^{12}$. Ce poème prédit la mort du tyran anglais et la libération imminente du peuple canadien.

Ces quatre poèmes politiques expriment des idées très voisines et reprennent parfois les mêmes images. L'Angleterre y est accusée de tyrannie, bien que cette tyrannie décroisse et se meure. Pour sa part, le jeune peuple canadien prend conscience de sa force grandissante:

10 Daniel Tracey, rédacteur de Vindicator, l'emporte alors sur Stanley Bagg, homme d'affaires en vue de Montréal, désigné par la Minerve comme le candidat de la «Bureaucratie»; il y a combats à coups de pierres entre les partisans des deux candidats; une troupe, qui se tient avec les partisans de Bagg, fait feu et tue trois citoyens: Casimir Chauvin, 20 ans, Languedoc et Billet (ou Billiette), deux citoyens de près de 60 ans; à la suite de ce triple meurtre, le colonel McIntosh et le capitaine Temple sont arrêtés pour meurtres volontaires, mais ils sont acquittés, au grand mécontentement de la population. (Feuilleton extraordinaire de la Minerve, 22 mai 1832; le Canadien, 23 et 30 mai 1832).

${ }_{11}$ Dans J. Huston, éd., op. cit., $2^{\mathrm{e}}$ éd., I: 376.

12 La Minerve, 20 octobre 1834; le Canadien, 22 octobre; reproduit dans J. Huston, op. cit., $2^{\mathrm{e}}$ éd., I: 280 . 
(...) la tyrannie

S'agite d'un puissant effort?

C'est sa convulsive agonie,

L'avant-courrière de sa mort.

L'Anniversaire du grand meurtre

Quand, noble, il jure avec fierté

D'entrer dans la nuit de la tombe,

Ou de ravir sa liberté?

\section{Le retour}

Turcotte juge le moment venu de lancer un appel à la révolution. Là perce son admiration pour les États-Unis, berceau de la démocratie occidentale. En cela, il suit son maître Papineau, qui est un fervent admirateur des institutions des États-Unis.

Un peuple doit choisir quand il voit réunis

La hache de la Pologne et les fers de l'Irlande,

Et qu'il voit la guirlande

Qui pend au front sacré de nos États-Unis.

Poème sans titre

Dis, dis d'une voix de tonnerre

Â ces tyrans audacieux:

Le lion règne sur la terre,

Mais l'aigle s'approche des cieux...

L'Anniversaire du grand meurtre

Nous, nous fixâmes notre étoile,

L'astre du peuple canadien:

Et l'ange à figure connue,

Planait au-dessus de la nue

Pour nous montrer la liberté...

Le retour

Peuples, vous êtes souverains!

Hommes, vous êtes tous égaux!

Un peuple est le maître de soi.

(...) les armes peuvent reprendre

Des droits par les armes acquis?... À l'Hon. L. J. Papineau

Écrire des poèmes d'inspiration politique et révolutionnaire n'est pas exceptionnel au cours des années 1830. Portant un jugement sur la littérature canadienne de cette période, Adolphe-Basile Routhier écrit:

Presque tous les poètes de ce temps-là débutaient par des chants patriotiques (...). C'est le ton général des poésies publiées de 1834 à 1837. Ce n'est plus seulement une ardeur patriotique qui les échauffe, c'est un souffle de révolte qui les anime. Les coeurs les 
plus jeunes ne chantent plus l'amour, mais la liberté. Ce nom magique absorbe tout, retentit partout. ${ }^{13}$

Chez tous les poètes, on trouve, dans les mêmes images et les mêmes symboles, le même mépris pour l'Angleterre ${ }^{14}$. Aux plaintes succèdent les mêmes réclamations, répétées comme un refrain: droits, réforme, liberté, justice, égalité ${ }^{15}$. À tous, la libération paraît imminente ${ }^{16}$. Mais si Turcotte partage son espérance avec les poètes de sa génération, il semble cependant se distinguer par ses propos séditieux. De tous les poèmes qui ont été publiés dans le Répertoire national, ceux de Turcotte sont les plus violents. Ils révèlent un Patriote radical ${ }^{17}$.

La philosophie libérale et révolutionnaire que Turcotte exprime dans ses poèmes est encore professée dans une lettre ouverte qu'il publie dans la Minerve du 12 février 1835. Cette lettre fait partie d'une polémique suivie dans les journaux du temps. Le 3 janvier précédent, un article du journal l'Ami du peuple, signé Un du clergé, posait cette question: «Dans les circonstances politiques actuelles, le clergé canadien doit-il demeurer passif ou, s'il doit agir, quelle doit être sa conduite, en général?» Les circonstances politiques auxquelles il est fait allusion, c'est l'affrontement maintenant irréductible entre la Chambre d'Assemblée et l'Exécutif, à propos de leurs droits constitutionnels respectifs. Dans cet affrontement, la Chambre refuse d'accorder les subsides au gouvernement, elle réclame un gouvernement responsable et un conseil législatif électif.

À la question posée, un correspondant de l'Ami du Peuple qui signe Franc-Patriote répond le 17 janvier. Il se montre en faveur de l'intervention occasionnelle du clergé dans la politique: le clergé n'estil pas pour le peuple le «berger du troupeau» ou «la sentinelle chargée de donner l'alarme?» De même qu'un prêtre doit révéler, s'il le connaît, un complot contre l'État, le prêtre qui jugerait un candidat «dangereux» pour l'ordre public doit s'y opposer par tous les «moyens constitutionnels» possibles. Puis Franc-Patriote s'oppose à l'esprit de rébellion du

\footnotetext{
13 Adolphe-Basile Routhier, dans J. Huston, éd., op. cit., $2^{\mathrm{e}}$ éd., I: XXX-XXXI.

14 Voir un poème anonyme de 1831 , «Plaintes et espoir», dans J. Huston, op . cit., $2^{\mathrm{e}}$ éd., I: 223-224; et un poème de 1834, «Pourquoi désespérer», de François-Xavier Garneau dans ibid.: 275-276.

15 Voir Napoléon Aubin, «Couplets en l'honneur de la Saint-Jean-Baptiste», 1835, dans ibid.: 301-303 et un poème anonyme, «Réforme et liberté», 1836, dans ibid.: 382-384.

${ }_{16}$ Voir François-Xavier Garneau, «L'an 1834», dans ibid.: 274-275; George-Étienne Cartier, «Ô Canada! Mon pays! Mes amours!», 1835, dans ibid.: 354-355 et François-Réal Angers, «L'avenir», 1836, dans ibid.: 383-385.

17 Un seul le rejoint dans ses chants révolutionnaires, Napoléon Aubin qui, dans une chanson de 1834, «Les Français aux Canadiens», écrit:

Le temps n'est plus, où le coeur en silence

Pouvait se taire au nom de liberté

Au noble sang qui coule dans vos veines,

ibid.: 287.

Ah! Canadiens, ah! ne résistez plus
} 
moment en invoquant le passage classique de saint Paul: «... que toute personne soit soumise aux puissances supérieures: car il n'y a point de puissance qui ne vienne de Dieu.» ${ }^{18}$ Cette opinion s'inspire de la plus pure tradition de l'Église catholique romaine, pour qui toute autorité est de droit divin. Et Turcotte, qui va exprimer une opinion différente, ne manque pas de découvrir un prêtre dans Franc-Patriote.

Dans la Minerve du 12 février suivant, paraît la lettre intitulée «Le clergé canadien et la politique du jour», signée des initiales J.E.T., qui ne peuvent prétendre cacher l'identité de Turcotte. Celui-ci s'en prend d'abord à l'argument central de Franc-Patriote, c'est-à-dire au caractère divin indéfectible de toute autorité et à une interprétation absolue de la pensée de saint Paul sur le sujet. Après avoir rappelé l'exemple des peuples qui ont trouvé bon d'abattre l'autorité des tyrans tels que les Néron et les Dioclétien et l'exemple plus lointain encore dans le temps, du peuple d'Israël lui-même, l'élu de Dieu, qui n'a pas hésité à prendre les armes pour se libérer de ses dominateurs, il s'attaque au principe qui interdit en conscience de détruire un pouvoir, ou même de lui résister. Si ce principe était vrai, dit-il,

...la lumière de lumière aurait apporté sur la terre des principes tendant à enchaîner la civilisation des peuples; car cette civilisation ne se perfectionne que par le perfectionnement progressif des liens de leur sociabilité qui ne sont autre chose que leurs gouvernements respectifs...

Développant ensuite la question de la source du pouvoir et de la légitimité de la révolution, il se montre partisan du libéralisme doctrinaire qui reprend, dans les années 1830, la pensée sociale des Philosophes du XVIII ${ }^{e}$ siècle. Il n'existe pas, prétend-il, de pouvoir sans peuple pour le créer, comme il n'existe pas d'enfant sans père. C'est donc le peuple qui est la source primitive, la source naturelle et essentielle du pouvoir et c'est au peuple, en tant que collectivité, qu'appartient le droit de changer les modalités du pouvoir:

Ce signe sensible de la volonté de Dieu [le peuple], cette source primitive du pouvoir, peut quand bon lui semble, concentrer sa force dans un mode quelconque de gouvernement ou de puissance; puis elle peut modifier ou changer entièrement cette puissance, quand son état de société subit lui-même des modifications qui requêrent [sic] des changements: et c'est à une telle puissance ainsi créée ou modifiée qu'il est défendu à chaque individu en particulier de résister, c'est en ce sens qu'aujourd'hui tous les peuples chrétiens entendent les paroles de saint Paul.

Par cette interprétation habile des paroles empruntées à saint Paul, il détruit l'argumentation de Franc-Patriote qui s'appuyait sur le même

${ }^{18}$ Épître de Paul aux Romains, chap. 13, v. 1. 
extrait de l'Épître aux Romains. Il termine enfin cette première partie de sa lettre en réfutant un autre argument utilisé par Franc-Patriote. À l'appui de son option conservatrice, celui-ci a évoqué les horreurs auxquelles ont donné lieu les révolutions européennes, particulièrement la Révolution française et l'Insurrection polonaise de 1830. Turcotte annule cette preuve en citant l'exemple des États-Unis d'Amérique:

Pourquoi ne pas plutôt porter vos regards et les nôtres du côté de nos voisins les Etats-Unis, et de tant d'autres jolies républiques, où le sang des victimes de la liberté n'a pas grossi les rivières ni même les ruisseaux? Vous dites: «Canadiens! regardez la France!» et moi je dis, pour contrebalancer vos paroles: Canadiens! regardez plus près de vous, regardez les États-Unis!!...

Puis, dans une deuxième partie de la lettre, répondant directement à l'essentiel de la question posée par Un du clergé, à savoir: «Dans les circonstances politiques actuelles, le clergé Canadien doit-il demeurer passif...?», Turcotte s'oppose à toute intervention du clergé en matière politique. Il appuie d'abord son opinion sur des citations scripturaires: "lls ne sont pas du monde comme je ne suis pas moi-même du monde»; ${ }^{19}$ et «Il ne contestera point, ni ne criera point, et personne n'entendra sa voix dans les places publiques» ${ }^{20}$. Il soulève ensuite trois objections d'ordre pratique. D'abord, le peuple ne saurait pas distinguer l'influence du prêtre en tant que prêtre de celle du prêtre en tant que simple citoyen, et l'influence du prêtre deviendrait vite indue, le caractère clérical donnant à cette influence une force plus grande dans un domaine étranger à la religion, l'ordre civil. En second lieu, il est peu probable que le clergé parvienne à l'unanimité sur cette question, d'où le risque pour celui-ci d'apparaître divisé aux yeux du peuple et de perdre du prestige. Enfin, dans l'hypothèse où le peuple canadien serait unanime à vouloir un changement de gouvernement, et le clergé, dans son ensemble, favorable au gouvernement britannique, le clergé apparaîtrait comme opposé au peuple. À la suite de tels arguments, Turcotte pose une conclusion très ferme: «...quelque [sic] soient les événements qui se préparent ici, le clergé ne devrait dans aucun cas, (m'entendezvous?) dans aucun cas quelconque, y intervenir...»

Les idées libérales et démocratiques que soutient Turcotte dans sa lettre à la Minerve, ce sont celles du temps. Aux dires d'un critique des lettres canadiennes, la période est «fertile en divagations démocratiques à Québec, à Montréal et jusqu'à la Baie Saint-Paul» ${ }^{21}$. Ce sont celles surtout des Patriotes. Celui qui est chef des Patriotes et leur maître à penser, Papineau, soutient dans un discours aux électeurs de Montréal

$19 \quad$ Évangile de Jean, chap. 17.

20 Évangile de Matthieu, chap. 12. 58.

21 Séraphin Marion, Les lettres canadiennes d'autrefois (Hull, Éd. de l'Éclair, 1942), III: 
en 1834: «Le peuple ne veut plus du système actuel...; il doit être changé à sa demande. ${ }^{22} \mathrm{Au}$ printemps de 1834, un correspondant d'un journal bas-canadien s'écrie: «Vive la souveraineté du peuple.» ${ }^{23} \mathrm{Au}$ banquet de la Saint-Jean-Baptiste, le 30 juin 1835, Denis-Benjamin Viger prononce un discours sur «le peuple, source légitime de tout pouvoir politique» ${ }^{24}$. Papineau et ses disciples se montrent également républicains et fervents admirateurs des institutions américaines qu'ils ne cessent de prôner comme modèles:

De tous ces gouvernements, écrit Papineau, ceux dont le régime a sans comparaison produit les plus heureux fruits, a été le républicanisme pur ou très légèrement modifié des états confédérés de la Nouvelle-Angleterre. ${ }^{25}$

À un autre moment, Papineau «célèbre avec un lyrisme un peu lourd», selon l'expression de Groulx, «le paradis américain».

Nous vivons et mourrons dans un pays placé en juxtaposition avec les États-Unis qui étalent aux yeux le spectacle instructif d'institutions démocratiques, occasionnant l'accroissement des richesses, donnant le bonheur, assurant les progrès du peuple, hâtant les plus rapides développements dont ait jamais été têmoin une société humaine. ${ }^{26}$

Dans son admiration pour le régime républicain, Turcotte apparaît donc comme l'émule de son chef.

Enfin, en refusant au clergé le droit d'intervenir en politique, Turcotte adhère indirectement au principe de la séparation de l'Église et de l'État, et ce principe est encore un principe cher à Papineau et à ses disciples. On se rappelle comment, à la session de 1831, Papineau a appuyé fortement le «bill des notables», qui était présenté par Louis Bourdages et qui devait permettre à presque tous les habitants d'une paroisse de participer à l'administration de la fabrique. On se rappelle en quels termes il a dénoncé l'attitude des membres du clergé qui, ditil,

prétendent former dans l'État, dans la Société civile, au milieu des citoyens tous assujettis aux lois, un ordre privilégié, indépendant [...] et ordonner comment seraient employés une partie des fruits du travail des Citoyens. ${ }^{27}$

C'est donc une idéologie «papineauiste» que partage Turcotte.

\footnotetext{
22 Dans John Hare, Les Patriotes, 1830-1839 (Montréal, Éd. Libération, 1971), 56-57.

23 Cité par Séraphin Marion, op. cit.: 58.

24 Ibid.: 59-60.

25 Fernand Ouellet, Papineau, Textes choisis (Québec, PUL, 1958), 53.

26 Lionel Groulx, Histoire du Canada français depuis la découverte (4 éd., Montréal, Fides, 1960), II: 141.

27 Fernand Ouellet, «Louis-Joseph Papineau» dans DBC, X: 624.
} 
Mais cette idéologie «patriote» n'est pas le fruit d'une génération spontanée au Canada français. Elle est née du mariage des théories politiques françaises du XVIII ${ }^{\mathrm{e}}$ siècle et du début du XIX ${ }^{\mathrm{e}}$ et de la pratique politique américaine, particulièrement de la démocratie jacksonnienne. Turcotte ainsi que ses maîtres connaissent Montesquieu, Voltaire et Rousseau. Ils empruntent à ce dernier sa thèse du Contrat social: le peuple est souverain, il s'est constitué librement par un pacte social et les citoyens ne sont plus tenus d'obéir lorsque ce libre pacte est rompu ${ }^{28}$. Mais ils ont aussi lu Lamennais dans ses Paroles d'un Croyant qui font l'objet de douze éditions locales, faites par les soins de Ludger Duvernay entre 1834 et 1837 et dont le Canadien et la Minerve reproduisent de larges extraits dans leurs colonnes. Chez Lamennais, Papineau et ses disciples ont trouvé la loi de l'égalité des individus: «Dieu n'a fait ni petits ni grands, ni maîtres ni esclaves, ni rois ni sujets: il a fait tous les hommes égaux... ${ }^{29}$ Ils y ont aussi trouvé une formulation plus radicale que chez Rousseau des principes démocrate et révolutionnaire:

Dans la balance du droit éternel, votre volonté pèse plus que la volonté des rois: car ce sont les peuples qui font les rois, et les rois sont faits pour les peuples, et les peuples ne sont pas faits pour les rois. Le père céleste n'a point formé les membres de ses enfants pour qu'ils fussent brisés par des fers, ni leur âme pour qu'elle fût meurtrie par la servitude... Il s'en trouva [des rois] [...] qui voulurent régner par eux-mêmes, comme s'ils eussent été d'une nature plus élevée que celle de leurs frères. Et le pouvoir de ceux-ci n'est pas légitime, car c'est le pouvoir de Satan, et leur domination est celle de l'orgueil et de la convoitise. Et c'est pourquoi, lorsqu'on n'a pas à craindre qu'il en résulte plus de mal, chacun peut et quelquefois doit en conscience leur résister... ${ }^{30}$

Cette influence idéologique subie indubitablement au Canada par la génération des années 1830, Groulx en mesure l'importance quand il écrit:

Les approches de l'insurrection nous font assister [...] à un singulier brassement de doctrines suspectes, d'illusions libérales, d'erreurs de toute espèce sur les notions de liberté, sur l'origine du pouvoir civil, sur les limites du pouvoir religieux. Les journaux des patriotes publient, avec une complaisante abondance, les discours des congressistes américains de 1776. Dans leurs écrits ou brochures, ils citent, comme des oracles, avec une emphase enfantine, Rousseau, Lamennais, Benjamin Constant. Les origines du mal se retracent néanmoins beaucoup plus haut. Remontons sans crainte jusqu'au dix-huitième siècle. Voyons-y un relent de l'En-

\footnotetext{
28 J.-J. Rousseau, Du contrat social (Paris, Garnier-Flammarion, 1966), 53-55, 125-128.

29 Félicité de la Mennais, Paroles d'un croyant (Paris, Daubrée et Cailleux, 18361837), 24.

30 Ibid., 72.
} 
cyclopédie beaucoup trop lue chez nous par ces têtes fortes qui sont d'ordinaire des têtes faibles... 31

On peut donc affirmer que, au grand déplaisir des autorités religieuses du temps, les Patriotes se plaisent dans la compagnie des libéraux français et que leur pensée en est profondément marquée ${ }^{32}$.

Loin de faire exception, Joseph-Édouard Turcotte se présente donc comme le type du jeune Patriote ${ }^{33}$. Dans les années 1830 , il est dans la vingtaine, il a été formé dans un séminaire, il se prépare à exercer une profession libérale, il est pécuniairement démuni, il s'intéresse à la politique, il a beaucoup lu, même ces oeuvres considérées comme pernicieuses par la hiérarchie catholique, et il écrit. Ces idées qu'il répand par la plume, ce sont celles de la philosophie libérale française, adaptée à la situation canadienne des années 1830, à la frustration économique que l'on ressent, à l'impasse constitutionnelle à laquelle on s'affronte, au nationalisme et à l'indépendantisme naissants. Ces idées, Turcotte les colore de sa verve, de sa fougue, de son radicalisme, de l'audace de ses vingt ans. Il ne s'en tient cependant pas à des publications éparses, poèmes de jeunesse et lettre ouverte d'un citoyen à son journal; il passe à l'action.

En février 1835, il pose sa candidature à l'élection complémentaire, dans la circonscription de Nicolet. Il s'agit de remplacer le vieux Patriote Louis Bourdages, un des fondateurs $d u$ Canadien, longtemps leader du parti canadien-français en Chambre puis bras-droit de Papineau et doyen de la Chambre, qui est décédé subitement le 20 janvier 1835. C'est à un homme d'une telle envergure que Turcotte ambitionne de succéder et il a l'appui d'un certain nombre de Patriotes. Il s'oppose à Jean-Baptiste Hébert, agriculteur, architecte et constructeur d'églises, marchand et major de milice de Saint-Grégoire de Nicolet, député de Nicolet de 1808 à 1814 , Patriote lui aussi ${ }^{34}$. Turcotte est défait par ce

\footnotetext{
31 Lionel Groulx, "La situation religieuse au Canada français vers 1840», dans Rapport de la Société canadienne d' histoire de l'Église catholique, 1941-1942: 57.

32 La période précédente, qui s'étend de 1760 à 1830 , est déjà marquée par l'incrédulité et l'esprit voltairien. Livres et journaux de France pénètrent au fond des malles, une petite école voltairienne se forme autour de la Gazette littéraire de Montréal, la bibliothèque publique de Québec, fondée en 1779, compte à ses débuts 40 livres de Voltaire, 23 de Rousseau et 35 de l'Encyclopédie. Les autorités religieuses s'en plaignent amèrement et admonestent leurs ouailles... Dans son Rapport au Saint-Siège, en 1794, Mgr Hubert peut déjà noter: «Au rapport des étrangers, elle [la corruption des moeurs] n'est pas si avancée dans beaucoup de grandes villes d'Europe.» Marcel Trudel, L'influence de Voltaire au Canada (Montréal, Fides, 1945), 42; Lionel Groulx, Notre maître, le passé, $2^{\mathrm{e}}$ série (Montréal, Granger, 1936): 172-177.

${ }_{33} \mathrm{~J}$. Huston, op. cit., $2^{\mathrm{e}}$ éd., I: 281 , prétend que Turcotte prend aussi part à la rédaction du Libéral, qui véhicule les idées révolutionnaires de juin à novembre 1837, qu'il en est même «l'un des rédacteurs les plus véhéments». Mais les articles du Libéral ne sont pas signés et le nom de Turcotte n'apparaît pas sur les deux listes de directeurs du journal, publiées par le journal adverse le Populaire, les 20 octobre et 10 novembre 1837. Ces deux faits ne contredisent cependant pas l'affirmation de Huston, et les écrits antérieurs de Turcotte semblent lui donner raison, même si nous ne sommes pas en mesure de sanctionner son jugement.

34 Jean-Baptiste Hébert sera emprisonné en 1837.
} 
candidat d'expérience. Il retourne à la vie privée et achève sa «cléricature».

Les débats pré-révolutionnaires de l'été et de l'automne 1837 permettent à Turcotte de se lancer à nouveau dans l'action. A la suite des Résolutions Russell de mars 1837 qui font fi de la Chambre d'Assemblée du Bas-Canada, le ton monte et le discours devient nettement révolutionnaire comme à l'assemblée des Six Comtés tenue à Saint-Charles en octobre 1837. Dans ce contexte, toute élection oppose non seulement Patriotes et partisans du gouverneur, mais Patriotes radicaux et Patriotes modérés. C'est le cas à l'élection dans la Basse-Ville de Québec en juin 1837. Turcotte appuie le Patriote radical Michael Connolly contre John Munn, entrepreneur en construction navale, et s'en prend violemment à l'ancien député de la circonscription, George Vanfelson, chef des Patriotes modérés depuis 1836, en remplacement de Bédard. À une assemblée tenue à la porte de l'église Saint-Roch, il monte sur une roue de charrette et harangue la foule en ces termes:

Que j' aurais désiré voir ici M. Vanfelson. Je suis jeune, messieurs, mais je suis Patriote, et je n'aurais pas craint de me mesurer avec lui, de le confondre. Il est singulier, messieurs, comme la petite famille fait des efforts pour élire un homme du goût de Gosford. Ne voyez-vous pas qu'elle agit ainsi en reconnaissance des dragées, sucreries, nanans, Champagne, qu'il leur a donnés? ${ }^{35}$

Le 3 septembre suivant, Turcotte participe à une autre assemblée publique, tenue à l'école des Glacis ${ }^{36}$. Cette assemblée a pour fin de former une association en vue de créer de l'emploi dans la construction navale, d'exercer une pression sur les banques pour obtenir la circulation des petites monnaies et surtout de tenter un rapprochement entre deux Patriotes en vue, le modéré Étienne Parent du journal le Canadien et le radical Robert-Shore-Milnes Bouchette du Libéral.

Turcotte participe ensuite à la formation du comité permanent de Québec mis sur pied le 17 septembre et créé sur le modèle du comité permanent de Montréal fondé en mai. Il est secrétaire de la réunion de fondation du comité qui se tient sur la place de l'église Saint-Roch ${ }^{37}$. Cependant, il n'est pas de ceux qui élaborent les principes politiques du comité au début d'octobre ${ }^{38}$.

C'est peut-être ce qui explique qu'aux premières heures des Troubles de 1837, il ne soit pas considéré par les autorités comme un Patriote de premier plan et soit laissé en liberté, alors que des membres

\footnotetext{
35 Le Canadien, 27 juin 1837. «La petite famille» est un terme péjoratif employé par les Patriotes radicaux pour désigner les modérés.

36 Lanadien, 4 et 6 sept.; la Gazette de Québec, 5 sept. 1837.

37 Le Populaire, 6 sept. 1837.

38 Les rédacteurs du manifeste sont Robert-Shore-Milnes Bouchette, Augustin-Norbert Morin, Charles Hunter et deux autres du nom de Voyer et de Quigley (Le Canadien, 9 octobre 1837).
} 
du comité permanent de Québec sont emprisonnés ${ }^{39}$. Laissé en liberté, Turcotte poursuit son action. Une fois l'avant-garde emprisonnée, il passe même en première ligne. Le 19 novembre, il prend part à une manifestation de plus de mille personnes; il harangue la foule qui est réunie au marché Saint-Paul et qui, de là, va pousser des hourras devant les résidences des prisonniers politiques ${ }^{40}$. Le Morning Herald du 20 novembre commente: «Le braillard Turcotte fit parler la sédition tout à son aise.» ${ }^{41}$

Mais c'est surtout par ses plaidoiries que Turcotte sert la cause des Patriotes. Ainsi, en décembre 1838, il obtient l'habeas corpus pour Célestin Houde, cultivateur de la Rivière-du-Loup (Louiseville), arrêté pour «manoeuvres sédicieuses». Il est alors complimenté par le juge Vallières de Saint-Réal pour la manière habile dont il lui a arraché la liberté de l'accusé ${ }^{42}$. Et, quelques semaines plus tard, il tente, en vain cette fois, d'obtenir l'habeas corpus en faveur de Joseph-Guillaume Barthe, accusé de haute trahison ${ }^{43}$.

Dans la suite, lorsque la Rébellion est complètement matée et que les chefs patriotes ont pris le chemin de l'exil ou la yoie du silence, Turcotte reste en contact avec Duvernay qui a fui aux États-Unis. Il est l'agent de la Minerve à Trois-Rivières où il vient de s'établir et il collecte les sommes dues à son $\mathrm{ami}^{44}$. Dans la correspondance échangée avec Duvernay, Turcotte se montre farouchement anti-britannique et confiant de voir triompher «la cause de la liberté». Il rapporte à Duvernay que se prépare, sous l'influence du clergé, une opposition à l'Union et une demande à l'effet de retourner à la constitution de 1791. Il désapprouve ce genre d'action; il y voit une trahison et il préfère, pour le moment, une espérance patiente:

Il y a des gens qui ont l'âme assez basse pour vouloir encore s'adresser à un gouvernement qui s'est abreuvé du sang de leurs concitoyens, sans parler de l'exil... Quant à ceux qui sont vraiment

\footnotetext{
39 C'est le cas, par exemple, de Pierre Chasseur, sculpteur de la rue Sainte-Hélène, chez qui se tenaient les séances du comité, arrêté le 11 novembre et remis en liberté sous cautionnement le 18; de Barthélémi Lachance, emprisonné le 13; de Joseph Légaré fils, peintre, propriétaire et directeur du Libéral, emprisonné le 13; d'Eugène Trudeau, marchand, qui se constitue prisonnier le 14; d'Augustin-Norbert Morin, arrêté le 15; de Robert-Shore-Milnes Bouchette, fondateur du Libéral et rédacteur de la section française de ce journal, accusé de haute trahison et emprisonné le 12 décembre; de Charles Hunter, rédacteur de la section anglaise du Libéral, qui est également accusé de haute trahison mais qui réussit à s'enfuir. (Aegidius Fauteux, Patriotes de 1837-1838 (Montréal, Éditions des Dix, 1950), 126, 172-173, 271, 295-296, 385-386; Antoine Roy, Les Patriotes de la région de Québec pendant la Rébellion de 1837-1838, dans les Cahiers des Dix, 24 (1959): 241-254.

40 Antoine Roy, loc. cit., 243.

41 Morning Herald, 20 nov., cité par le Populaire, 24 nov. 1837.

42 Aegidius Fauteux, op. cit., 269; Meinier (Napoléon Caron), dans l'Opinion publique, 11 déc. 1873,591 .

43 Joseph-Guillaume Barthe, Souvenirs d'un demi-siècle ou Mémoires pour servir à l' histoire contemporaine (Montréal, Chapleau, 1885), 393-398.

44 J.-E. Turcotte à lLudger Duvernay, 2 fév. 1840, ANQ, Papiers Duvernay, f. 405.
} 
pour la cause de la liberté de leur pays, ils dédaigneront de s'adresser au gouvernement anglais pour n'importe quelle demande et ils attendront en silence que la justice de leur cause et la providence viennent à leur secours... Adieu, ne vous découragez pas, espérez et croyez qu'il y a encore au Canada beaucoup, beaucoup de bons Canadiens à la tâche. ${ }^{45}$

Il convient ici de souligner que c'est à Ludger Duvernay, au fondateur de l'Association des Frères Chasseurs qui entretient les objectifs d'intervention militaire, que Turcotte écrit ces lignes. Ainsi, l'échec de la Rébellion, la répression qui a suivi et l'Union qui est votée, ne font que différer le rêve révolutionnaire, «indépendantiste» de Turcotte. L'image qu'il projette en 1840 demeure radicale. Mais, comme bien d'autres, il optera pour le compromis, une fois l'Union des Canadas réalisée ${ }^{46}$. Au printemps de 1841, il se présente comme candidat anti-unioniste, mais, dès l'automne suivant, il passe à la solde du gouvernement comme traducteur des lois. En 1844, il se range parmi les réformistes, mais à l'élection complémentaire de juin 1845 dans Trois-Rivières, il fait élire Viger, dont l'alliance avec les tories fait scandale; en 1847, il est solliciteur général dans le gouvernement Sherwood; et il s'oppose à LaFontaine aux élections qui précèdent la reconnaissance de la responsabilité ministérielle. Après la formation de l'alliance libérale-conservatrice, il devient un partisan de Cartier, qui en fait le président de la Chambre en mars 1862.

Voilà un Patriote «ordinaire», un Patriote ignoré, un Patriote oublié, comme il y en a des milliers dans l'histoire des Rébellions de 18371838. Sortir de l'ombre «la période patriote» de Joseph-Édouard Turcotte, c'est mettre l'accent sur les oublis et les oubliés d'une période pourtant très étudiée de notre histoire. Jusqu'ici, la caméra a été braquée sur les chefs et sur «les martyrs». Si on arrivait à identifier d'autres visages dans la foule qui a été rejointe par le mouvement patriote et entraînée dans la Rébellion, on aurait une connaissance plus concrète et plus complète du mouvement et de l'idéologie qui l'anime. L'histoire des Rébellions n'en serait sans doute pas refaite mais elle s'appuierait sur une base plus large. Et l'histoire sociale des années 1830 y trouverait son compte.

45 J.-É. Turcotte à Ludger Duvernay, 2 fév. 1840, ANQ, Papiers Duvernay, f. 383.

46 Voir Louisette Pothier, «Joseph-Edouard Turcotte», dans $D B C$, IX: 878-880. 